\title{
A bibliometric review of research trends in neuroimaging
}

\author{
Andy Wai Kan Yeung ${ }^{1, *}$, Tazuko K. Goto ${ }^{1,2}$ and W. Keung Leung ${ }^{3}$ \\ ${ }^{1}$ Oral and Maxillofacial Radiology, Applied Oral Sciences, Faculty of Dentistry, The University of Hong Kong, Hong Kong \\ ${ }^{2}$ Department of Oral and Maxillofacial Radiology, Tokyo Dental College, Tokyo, Japan \\ ${ }^{3}$ Peiodontology, Faculty of Dentistry, The University of Hong Kong, Hong Kong
}

\begin{abstract}
Neuroimaging is one of the important medical imaging domains that help diagnose and manage diseases. This study describes the neuroimaging publication outputs sorted by journals, countries, authors and institutions, and evaluates journal performance using metrics based on publication data from 2003 to 2014 indexed in the Web of Science and Journal Citation Reports. There has been a significant growth in the neuroimaging literature with North America and Europe being the main contributors. Magnetic resonance imaging is the most popular imaging modality, and brain connectivity is one of the hotspots. Top journals within the field have improved performances over the study period.
\end{abstract}

Keywords: Bibliometrics, impact factor, neuroimaging journals, research evaluation.

NeURoImAgING is one of the important medical imaging domains that help diagnose and manage diseases. For instance, magnetic resonance imaging (MRI) enables clinicians to examine the internal structure of patients' bodies and detect brain activities without the use of ionizing radiation $^{1,2}$. Neuroimaging researches have contributed to providing quality data to clinicians for making diagnoses and managing patients more efficiently.

However, have neuroimaging researches received their deserved attention and thus gained impact over time? Bibliometrics could provide an answer by applying quantitative analysis and statistics to describe trends of academic publications and their citation counts ${ }^{3}$. It allows us to map the overview of the specific literature, identify the most productive authors within the field and evaluate journal performances ${ }^{4}$. It has been extensively employed to evaluate research trends in relevant fields such as artificial blood vessels ${ }^{5}$, bioelectrochemistry ${ }^{6}$, materials science $^{7}$ and neuroscience ${ }^{8}$, with neuroimaging potentially standing in the middle of these fields with crossdisciplinary connections. Moreover, it has been reported that researches within the same field have received different extents of attention and thus the number of citations. For example, basic researches had a higher impact than clinical interventional researches ${ }^{9}$. Therefore, the

\footnotetext{
*For correspondence. (e-mail: ndyeung@hku.hk)
}

aim of this study is to describe the general landscape of the neuroimaging literature, evaluate performance trends of neuroimaging journals as a whole and to analyse four representative journals with the highest and lowest impact factors (IFs) within this category. These results could form the basis for better understanding of the global neuroimaging research.

\section{Methods \\ Source of data}

The study was based on data provided by the Web of Science (WoS) Core Collection and the Journal Citation Reports (JCR) Science Edition. Both are subscriptionbased and hosted by Thomson Reuters, which gives each journal an IF calculated by 'dividing the number of citations in the JCR year by the total number of articles published in the two previous years ${ }^{10}$. Besides IF, there is more bibliometric information disseminated by JCR that evaluates and compares the performance of each journal and each subject category from various perspectives. We searched the database for journals classified within the 'neuroimaging' category between 2003 and 2014. For the sake of simplicity, these journals would be referred as neuroimaging journals in this study. Year 2003 is the first year when journal category data became available, and such data have been updated up to year 2014.

\section{Bibliometric analysis of neuroimaging literature}

Full record and cited references from all articles and reviews published in neuroimaging journals between 2003 and 2014 were exported as text files from WoS. Publication counts were sorted by author, country/region, language, institution, research area and journal title. We identified, based on publication count, the top 10 authors, countries/regions and institutions. We examined the co-authorship network at the levels of country/region and institution. Also examined was the co-citation network to reveal the relevant research fields that produced publications commonly co-cited by neuroimaging publications. This would reveal the interdisciplinary directions of the neuroimaging field. Finally, based on the wordings of the 
titles and abstracts of these publications, we created a term map to illustrate the network of frequent keywords and thus the hotspots of this field. The inspection and visualization of networks were performed with VOSviewer $^{11}$.

To create co-authorship network maps, we first screened for countries/regions with more than 12 coauthorship counts within this period (on average one annual co-authorship with others). Among the included candidates, the top 25 with most co-authorship links to each other were selected, and their co-authorship links within this network were mapped. VOSviewer creates network maps using the VOS layout and VOS clustering techniques $^{12,13}$. As a result, countries/regions that coauthor with each other more frequently would be grouped into the same cluster and located closer to each other within the network map. The same procedures were repeated for institutions.

To create the co-citation map, all reference lists of the selected publications were examined. Only journals that received at least 50 citations were considered. Among these, the top 500 with most co-citation counts with each other were selected, and their co-citation links within this network were mapped. The journals were grouped into four clusters, with each cluster having at least 10 journals. Journals that were co-cited more frequently with each other would be grouped into the same cluster and located closer to each other within the map.

For the term map, the titles and abstracts of the publications were parsed to yield all noun phrases. VOSviewer incorporates an algorithm that enables the exclusion of general noun phrases that co-occur with many different noun phrases instead of being specifically related ${ }^{14}$. Only terms occurring in at least 50 publications were considered. The algorithm was run to produce 2000 terms. We checked the outcome list, and manually identified and removed irrelevant noun phrases such as background, information, introduction, purpose, method, procedure, material, result, discussion and conclusion ${ }^{15}$. Abbreviated forms of the noun phrases were combined. Similar to the network maps, the terms that co-occurred more (in terms of the number of publications) would be located closer to each other. The term map was coloured to illustrate the impact of the keywords by the relative citations received by them. To illustrate the relative citations, the citation count of each publication was normalized. Each term received a score based on the normalized citation counts of the publications in which it occurred. Colours ranged from blue to green to red, where blue indicated an impact lower than average, green indicated an averaged impact, while red indicated an impact higher than average.

The above-mentioned procedures have been described in detail and utilized to map the scientific literature on computer and information ethics ${ }^{15}$, patient safety ${ }^{16}$, and various medical fields ${ }^{9,17}$.
Bibliometric analysis of neuroimaging journals as a category

To examine the overall developmental trend of neuroimaging journals, we collected several categorical bibliometric indicators from the JCR. The indicators have been defined and released annually by Thomson Reuters ${ }^{10}$, and presented in a manner similar to that of Jayaratne and Zwahlen ${ }^{18}$.

1. Number of journals.

2. Publication frequency: annual, semi-annual, quarterly, bimonthly, monthly.

3. Total cites: 'the total number of times that (journals in the subject category) has been cited by all journals included in the database in the JCR year.'

4. Median impact factor: 'the median value of all journal Impact Factors in the subject category.'

5. Aggregate impact factor: 'calculated the same way as the Impact Factor for a journal, but it takes into account the number of citations to all journals in the category and the number of articles from all journals in the category.'

6. Aggregate immediacy index: 'the average number of times an article is cited in the year it is published.'

7. Aggregate cited half-life: 'the median age of the articles (within this subject category) that were cited in the JCR year. The aggregate cited half-life is an indication of the turnover rate of the body of work on a subject.'

8. Aggregate citing half-life: 'Is the median age of articles [regardless of subject category] cited by journal in this category in the JCR year.'

9. Number of articles, reviews and others (such as 'editorials, letters, news items, and meeting abstracts').

10. Number of references per publication count for articles, reviews and others.

\section{Bibliometric analysis of representative neuroimaging journals}

Jayaratne and Zwahlen ${ }^{18}$ used IF in 2003 to identify the top five and bottom five dental journals as representatives of the 46 dental journals, and track their performances over the study period $(10 / 46=21.7 \%)$. Among the 13 journals in the 'neuroimaging' category listed in JCR 2003, we maintained a similar proportion of journals as representatives and thus identified two with the highest IF and two with the lowest IF $(4 / 13=30.8 \%)$. To trace their development over the period 2003-2014, we retrieved annually their IF, eigenfactor score, immediacy index and total cites. Eigenfactor score is available for individual journals, but not subject category. It is 'available only for JCR years 2007 and later' and 'based on the number of times articles from the journal published in the past five years have been cited in the JCR year' ${ }^{, 10}$. 
Further, by considering 'which journals have contributed these citations', eigenfactor score is uninfluenced by selfcitations. It will be higher if the articles are cited by more highly cited journals. In 2003, Rivista Di Neuroradiologia had the lowest IF 0.152; it was discontinued and renamed in June 2006, and thus was excluded from this study.

We assessed the time trends of the neuroimaging journal category and representative journals. Linear regression model was used for each bibliometric indicator, in which the dependent variable was the indicator and the independent variable was the year ${ }^{18-20}$. The slope of the regression, $\beta$, indicates how on an average the value of the indicator will change within one year. The coefficient of determination, $R^{2}$, indicates how well the model can explain the variation.

To assess if the total number of citable items (articles and reviews) of the neuroimaging journal category was correlated with its bibliometric indicators, Pearson correlation was used to test its correlation with total cites, median IF, aggregate IF and aggregate immediacy index ${ }^{18,21}$.

All statistical analyses were performed with SPSS 23.0 (IBM, New York, USA). Test results were considered as significant if $P<0.05$.

\section{Results}

\section{Distribution of neuroimaging publications}

Between 2003 and 2014, there were 23,155 articles and 1155 reviews published in the neuroimaging journals.
The top ten most productive authors, out of 56,090, were Thompson, Paul M. (158; 0.65\%), Toga, A. W. (147; $0.61 \%)$, Kallmes, D. F. (130; 0.54\%), Friston, K. J. (128; $0.53 \%)$, Calhoun, V. D. (102;0.42\%), Filippi, M. (90; $0.37 \%)$, Fox, P. T. (89; 0.37\%), Eickhoff, S. B. (78; $0.32 \%)$, Zilles, K. (77; 0.32\%) and Barkhof, F. (72; $0.30 \%$ ). The top ten countries/regions, out of 92 , were USA $(10,354 ; 42.59 \%)$, Germany $(3729 ; 15.34 \%)$, England $(2436 ; 10.02 \%)$, Japan $(1707 ; 7.02 \%)$, Canada (1487; 6.12\%), France (1475; 6.07\%), Italy (1272; $5.23 \%)$, The Netherlands (1207; 4.97\%), China (1019; $4.19 \%)$ and Switzerland $(887 ; 3.65 \%)$. Publications were predominately in English $(23,838 ; 98.06 \%)$, followed by German (295; 1.21\%), French (157; 0.65\%), Italian (24; $0.10 \%)$ and Romanian $(1 ;<0.01 \%)$.

Regarding international collaborations, the most collaborative countries/regions are located in North America and Europe (Figure 1), namely USA (3858; 20.4\%), Germany $(2361 ; 12.5 \%)$ and England (2178; 11.5\%), which are also the ones with highest number of publications. At the institutional level, collaborations were predominantly within the same country/region. In USA, the institution with highest co-authorship count was Harvard University $(666 ; 15.1 \%)$. In Germany, it was University of Düsseldorf $(51 ; 1.2 \%)$. In England, it was University College London (197; 2.2\%).

\section{Co-citation network of the neuroimaging literature}

Neuroimaging publications were interconnected to various relevant research fields which were identified

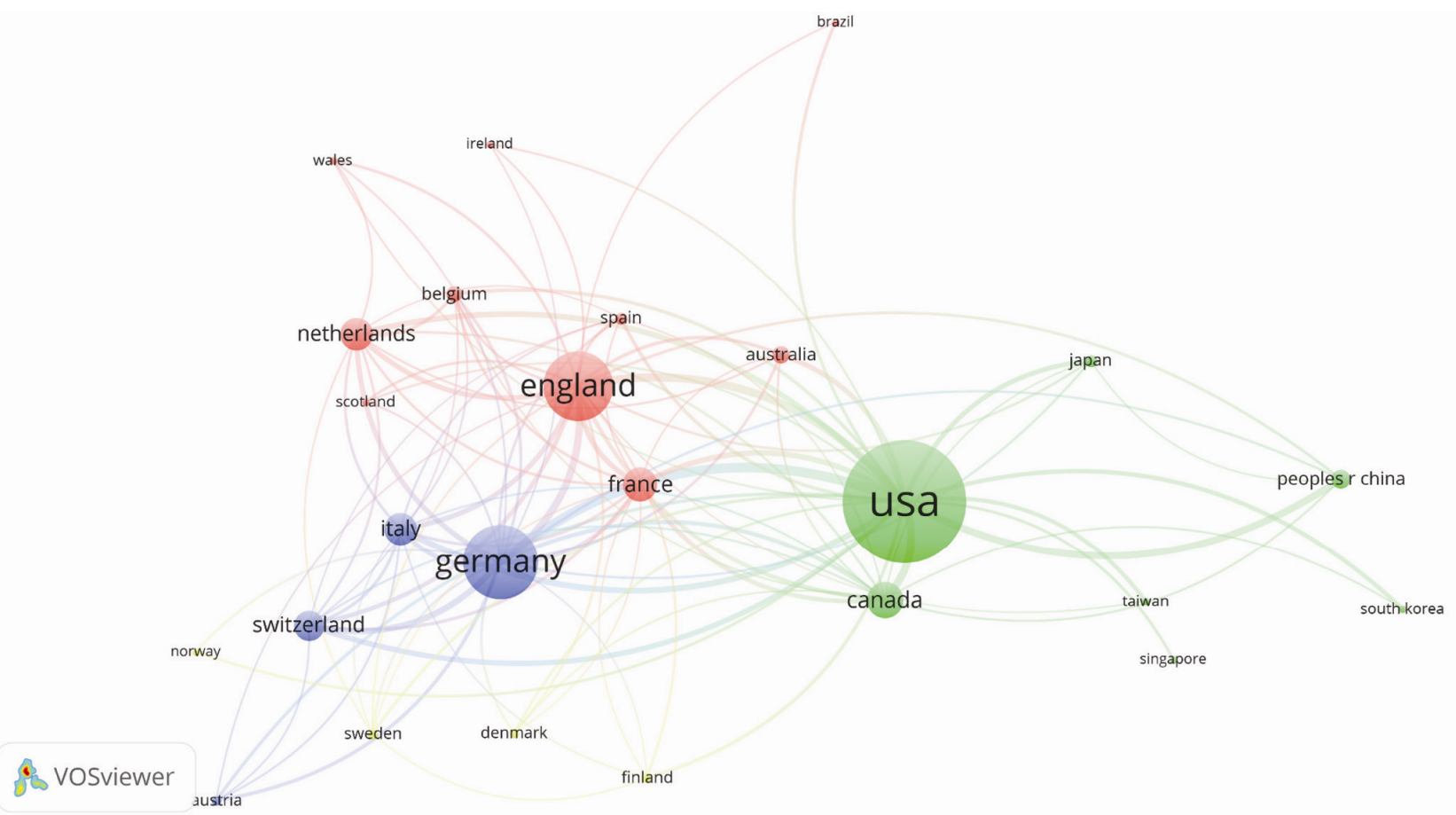

Figure 1. Co-authorship map showing the top 25 countries/regions with the highest number of co-authorships with one another. Each of them has at least 12 co-authorship counts and the top 100 co-authorship connections are shown. Circle size and line strength indicate the co-authorship count. 


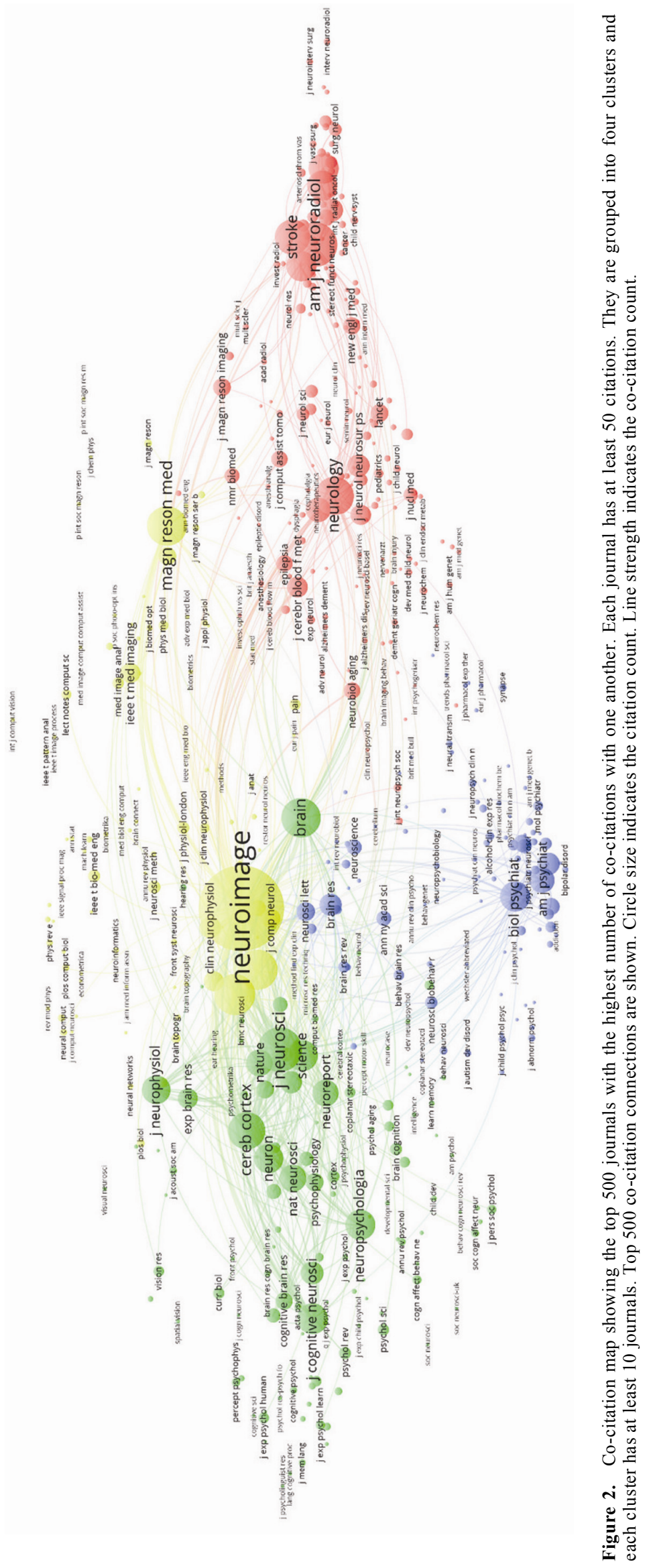


according to the JCR journal categories. As shown in Figure 2, Neurolmage is at the centre of the co-citation network. The various parts of the co-citation map are described below.

The red cluster contains journals mainly from clinical domains such as clinical neurology (e.g. Neurology, Stroke), general and internal medicine (e.g. New England Journal of Medicine, Lancet) and surgery (e.g. Journal of Neurosurgery). The yellow cluster contains journals mainly from engineering (e.g. IEEE Transactions on Biomedical Engineering), computer science (e.g. IEEE Transactions on Medical Imaging), and radiology, nuclear medicine and medical imaging (e.g. Magnetic Resonance in Medicine). The green cluster contains journals mainly from multidisciplinary sciences (e.g. Nature, Science) and neurosciences (e.g. Journal of Neuroscience, Journal of Cognitive Neuroscience). The blue cluster contains journals mainly from psychiatry (e.g. Biological Psychiatry, American Journal of Psychiatry).

\section{Term map of the neuroimaging literature}

Figure 3 shows a term map of the neuroimaging literature. The map shows a distinction between different research areas. The clinical research terms (e.g. aneurysm, haemorrhage, stenosis) are located mainly on the left, while the basic research terms (e.g. emotion, empathy, memory) are on the right. Moreover, the left side of the map is mainly coloured blue and green, while the right side is mainly coloured yellow, orange and red. This implies that the terms related to basic research areas have a higher citation impact in general compared to those related to clinical research areas. From the circle size, it can be seen that various MRI techniques (e.g. MRI, functional MRI (fMRI), diffusion tensor imaging (DTI), diffusion weighted imaging (DWI)) are the predominant imaging modalities compared to others such as computed tomography, positron emission tomography and electroencephalography. One of the hotspots of the field having highest citation impact is to study how various parts of the brain communicate with one another, as the terms brain network, network, connectivity, effective connectivity are all coloured red.

\section{Overall changes in the neuroimaging journals as a category}

Table 1 provides details of the overall changes in the neuroimaging journals. The total number of journals in the neuroimaging category of JCR is stable between 12 and 14 during the study period (Table 1). The number of journals within various publication frequencies did not change significantly $(P>0.05$, Figure $4 a)$. However, the total number of publications in these journals reached
2954 in 2014, which was 1.79 times the count in 2003 (Figure $4 b$ ). There was significant annual growth in the number of research articles $\left(\beta=85.157, R^{2}=0.824\right.$, $P<0.001)$ and reviews $\left(\beta=9.007, R^{2}=0.428, P=0.021\right)$. The 'neuroimaging' category also demonstrated a significant rise in the median IF $\left(\beta=0.115, R^{2}=0.747\right.$, $P<0.001), \quad$ aggregate IF $\quad\left(\beta=0.206, \quad R^{2}=0.925\right.$, $P<0.001$; Figure $4 c$ ) and aggregate immediacy index $\left(\beta=0.072, R^{2}=0.977, P<0.001\right)$.

Noticeably, the number of citations received by neuroimaging journals increased significantly over the years and quadrupled from 30,084 in 2003 to 131,513 in 2014 $\left(\beta=9159, R^{2}=0.982, P<0.001\right.$; Figure $\left.4 d\right)$. There were significant increases in the aggregate cited half-life $\left(\beta=0.167, R^{2}=0.959, P<0.001\right)$ and citing half-life $\left(\beta=0.107, R^{2}=0.957, P<0.001\right)$. In JCR 2014, the 'neuroimaging' category had a cited half-life of 6.5 years. It means that $50 \%$ of all neuroimaging journal articles cited by articles of the same category in 2014 were published within the 6.5 years immediately before the end of 2014. Compared with 4.9 in 2003, the increase in cited half-life indicates that neuroimaging articles in general became cited for a longer period over the years of development in this field. The number of references per publication significantly increased for research articles $\left(\beta=1.601, R^{2}=0.981, P<0.001\right)$, but declined for reviews $\left(\beta=-2.667, R^{2}=0.505, P=0.010\right)$.

The combined count of article and review correlated positively with the total cites $(r=0.942, P<0.001)$, median IF $(r=0.752, P=0.005)$, aggregate IF $(r=0.803$, $P=0.002)$ and aggregate immediacy index $(r=0.888$, $P<0.001)$.

\section{Changes in the representative neuroimaging journals}

In 2003, the top two neuroimaging journals with the highest IF were NeuroImage (6.192) and Human Brain Mapping (6.058, Figure 5). The two journals with lowest IF were Klinische Neurophysiologie (0.298) and Stereotactic and Functional Neurosurgery (0.425). Figure 5 shows the changes in their key publication metrics. Among these four journals, there were significant increases in the IF over the period 2003-2014 for NeuroImage $\left(\beta=0.081, R^{2}=0.44, P=0.019\right)$, Human Brain Mapping $\left(\beta=0.135, R^{2}=0.351, P=0.042\right)$ and Stereotactic and Functional Neurosurgery $(\beta=0.108$, $\left.R^{2}=0.670, P=0.001\right)$, but not for Klinische Neurophysiologie $\left(\beta=0.005, R^{2}=0.033, P=0.570\right)$.

Besides, there were similar significant increases in the eigenfactor score of NeuroImage $\left(\beta=0.003, R^{2}=0.585\right.$, $P<0.001)$ and Human Brain Mapping $(\beta=0.003$, $\left.R^{2}=0.938, P<0.001\right)$, but not Klinische Neurophysiologie $\left(\beta=0.000, R^{2}=0.018, P=0.749\right)$ or Stereotactic and Functional Neurosurgery $\left(\beta=0.000, R^{2}=0.109\right.$, $P=0.424)$. 


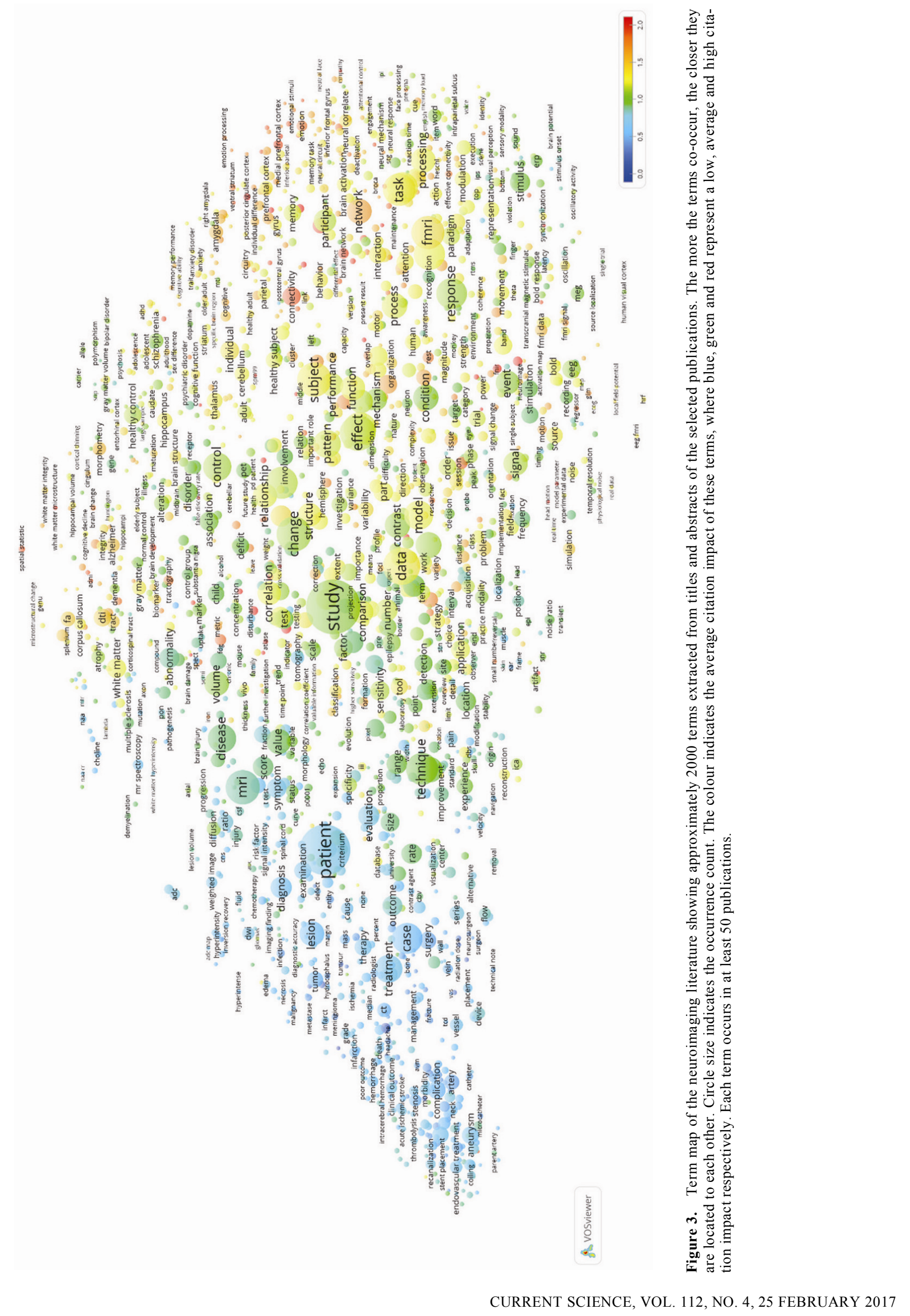




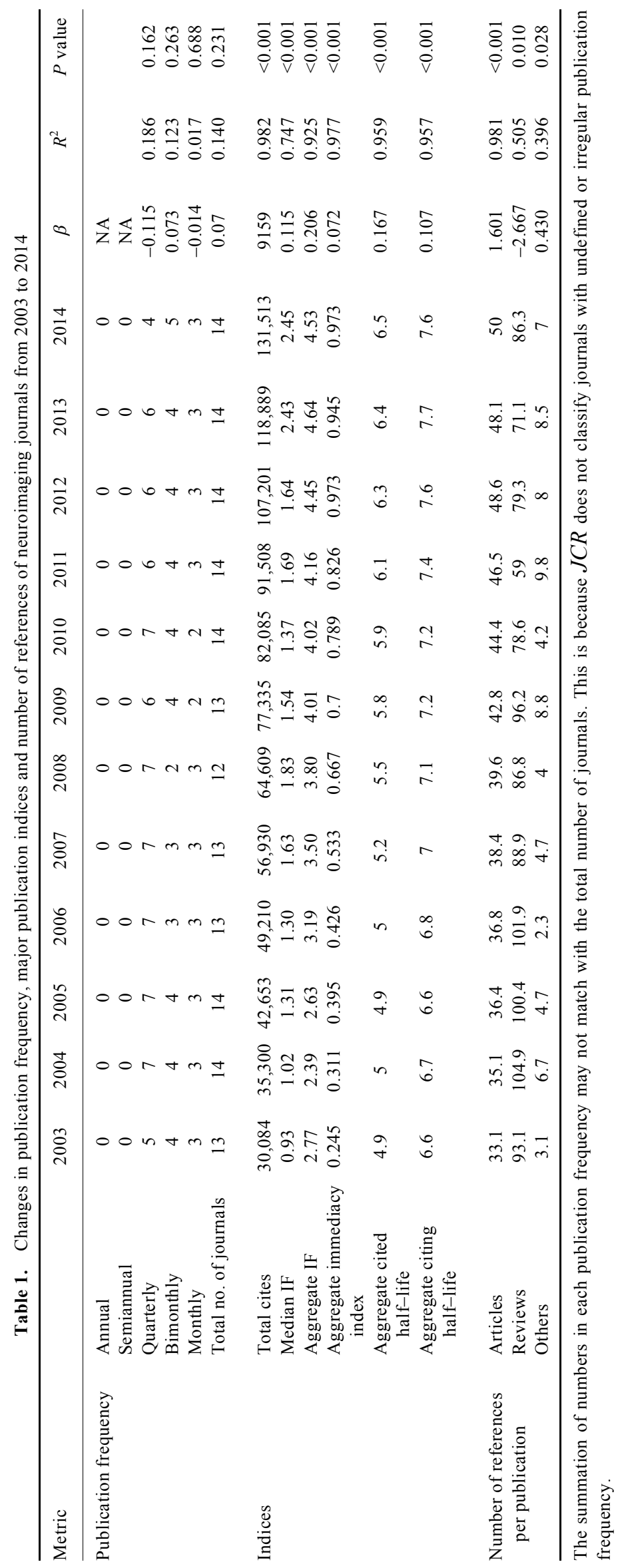



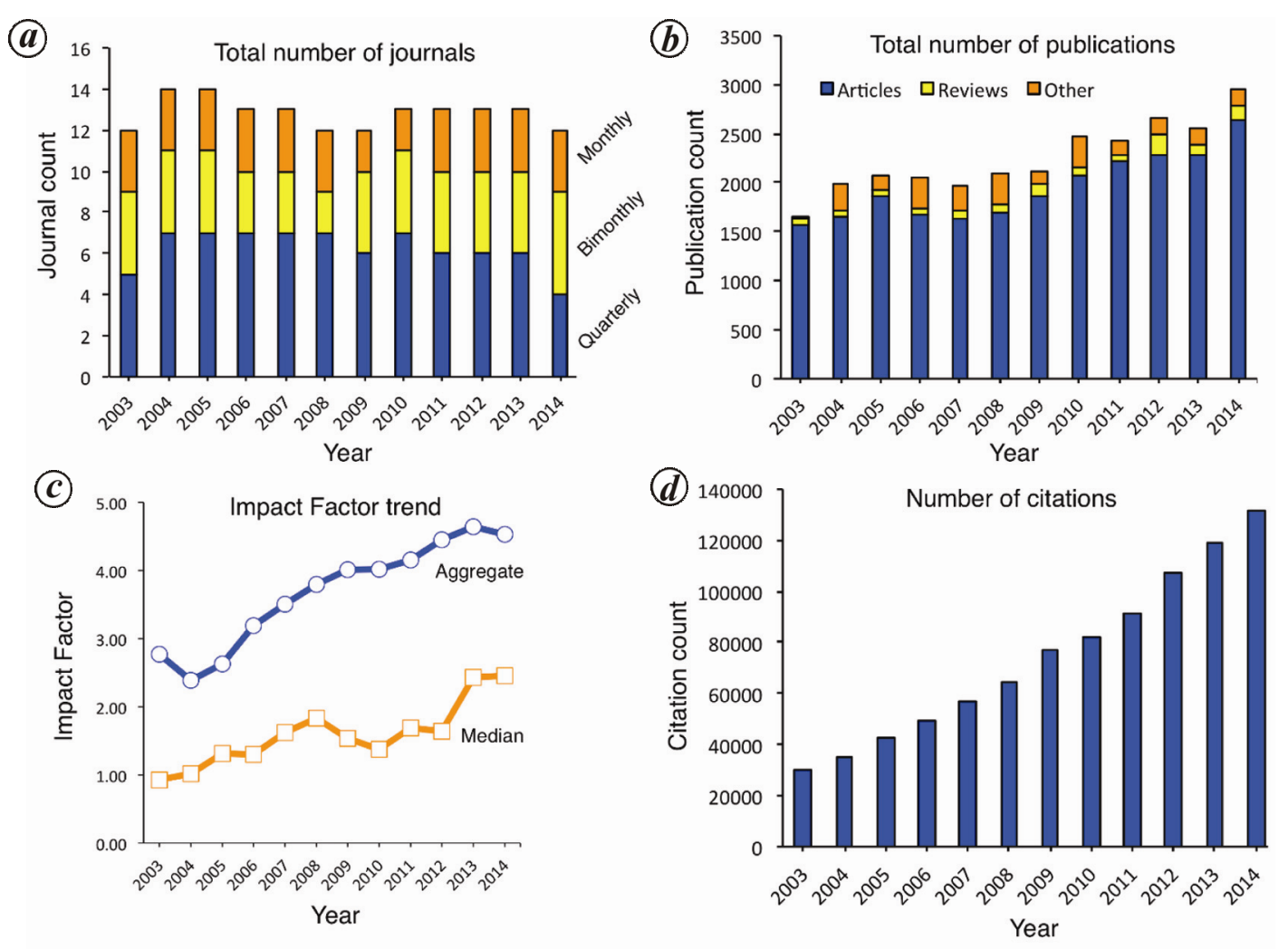

Figure 4. Time trends of key publication metrics of all neuroimaging journals as a whole from 2003 to 2014 . $\boldsymbol{a}$, Total number of journals; $\boldsymbol{b}$, total number of publications; $\boldsymbol{c}$, aggregate and median impact factors; $\boldsymbol{d}$, number of citations received.

In terms of a significant increase in the immediacy index, the journal with the best performance was NeuroImage $\left(\beta=0.100, R^{2}=0.976, P<0.001\right)$, followed by Human Brain Mapping $\left(\beta=0.050, R^{2}=0.477, P=0.013\right)$ and Stereotactic and Functional Neurosurgery $(\beta=0.021$, $\left.R^{2}=0.678, P=0.001\right)$. Klinische Neurophysiologie did not have a significant change in its immediacy index ( $\beta=0.005, R^{2}=0.225, P=0.119$ ).

NeuroImage also showed the highest increase in total cites $\left(\beta=6348.78, R^{2}=0.981, P<0.001\right)$. One noticeable point was that the total cites of NeuroImage accounted for only $30 \%$ of total cites of neuroimaging journals in 2003, but its share increased to more than $59 \%$ in 2014. There were also significant increases in total cites of Human Brain Mapping ( $\beta=1207.12$, $\left.R^{2}=0.948, P<0.001\right)$ and Stereotactic and Functional Neurosurgery $\left(\beta=53.96, R^{2}=0.682, P=0.001\right)$, but not for Klinische Neurophysiologie $\left(\beta=1.07, R^{2}=0.28\right.$, $P=0.077)$.

\section{Discussion}

JCR defines the 'neuroimaging' category as a subject that 'covers resources on the mapping technologies used to treat, diagnose, or monitor brain lesions and mental dis- orders $^{22}$. Thus, journals in this category are expected to have a broad range of readers from scientists to healthcare providers.

To the best of our knowledge, this study is the first to quantitatively evaluate the overall neuroimaging literature landscape as well as the academic performance of neuroimaging journals around the last decade. From the term map, we can demonstrate that terms related to basic research areas generally have a higher citation impact than those related to clinical interventions. This finding is consistent with previous studies that investigated medical fields of cardiac and cardiovascular systems, clinical neurology and surgery that were highly relevant ${ }^{9}$. While publications and collaborations mainly involved North America and Europe, MRI was the most popular neuroimaging modality to investigate various aspects of the brain, particularly the brain network/connectivity. The popularity of MRI may be attributed to its versatility, non-invasiveness and no radiation used. The extensive co-citation network implies that these researches are highly relevant to other fields such as clinical neurology, surgery, engineering, computer science, neurosciences ${ }^{23}$ and psychiatry.

We found that while the number of neuroimaging journals remained stable, all the bibliometric indices of the subject category demonstrated a significant increase over 

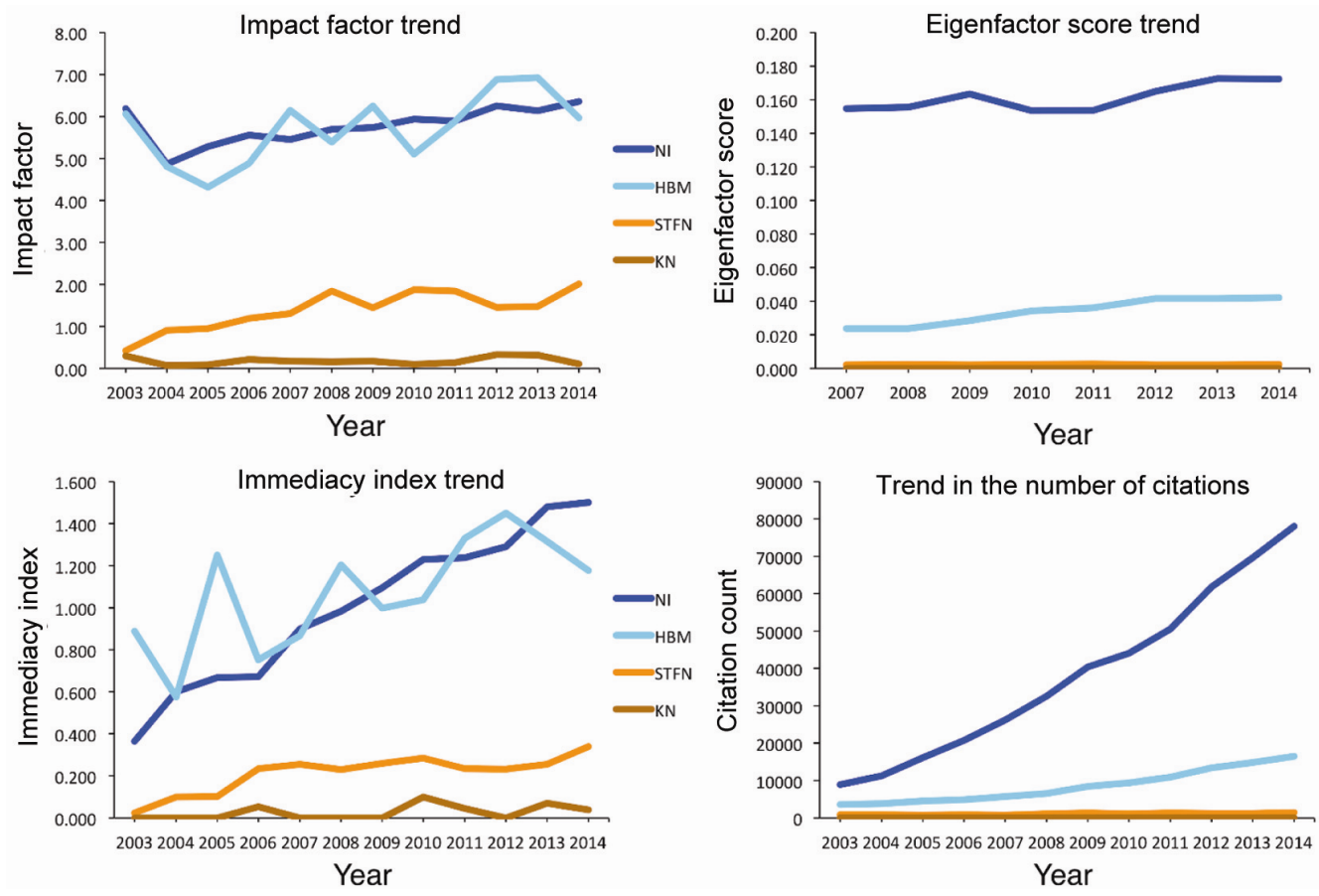

Figure 5. Time trends of key publication metrics of individual representative journals from 2003 to 2014. Eigenfactor score is only available in JCR since 2007. NI, NeuroImage; HBM, Human Brain Mapping; STFN, Stereotactic and Functional Neurosurgery and KN, Klinische Neurophysiologie.

the period. As IF has its own shortcomings ${ }^{24}$, this study has presented a series of other metrics also included by the $J C R$ to evaluate the performance of neuroimaging journals in a more comprehensive manner. Within the limitations of the study, we found that neuroimaging journals were getting more attention (increased total cites), having more impact (increased median and aggregate IFs), and having a faster and longer influence (increased immediacy index and aggregate cited half-life). These may be related to an increasing availability of the Internet and electronic version of publications that allow scholars to access materials in a faster and broader way ${ }^{18}$. Besides, the neuroimaging research field has been experiencing rapid growth as observed by a significant increase in the total number of publications reported in this study. It may also have contributed to the surge of the metrics as reflected by positive correlations between them.

We analysed individual neuroimaging journals that had the highest and lowest IFs back in 2003. We found that the best performing journals, namely NeuroImage and Human Brain Mapping, had a steady rise in their IF and eigenfactor score. Regarding journals at the other end, only Stereotactic and Functional Neurosurgery had an improvement in its IF. Also, Klinische Neurophysiologie and Stereotatic and Functional Neurosurgery showed no improvement in the eigenfactor score that omits the influence of self-citations.

Eigenfactor score measures the total importance of a journal to the scientific community, and its summation from all JCR-indexed journals equals 100 (ref. 25). This study reveals that the total importance of NeuroImage (0.153-0.172) has been consistently 4-6 times that of Human Brain Mapping (0.024-0.042) during the period 2007-2014. In 2014, NeuroImage published only twice as much as it did in 2003, but its number of total cites was nearly nine times what it was in 2003 . The growth in citation count by NeuroImage was much more than that of Human Brain Mapping, the second top neuroimaging journal in terms of IF.

This study has a number of limitations. First, we only analysed data from in WoS and $J C R$, and so publications in non-indexed neuroimaging journals have not been considered. Also, there might exist erroneous or missing data in the database that we would not be able to identify and rectify. Nonetheless, this study is useful as a reference for research evaluation by reviewers, hiring managers and grant panels ${ }^{26}$.

\section{Conclusion}

Bibliometric information provides scholars with useful indications about the current status of the neuroimaging research field and its key journals. After considering various metrics, it could be concluded that research publications relevant to the neuroimaging community have been expanding and gaining impact. MRI is the most popular imaging modality for research. Amongst all titles indexed in the 'neuroimaging' category in JCR, NeuroImage and Human Brain Mapping have been two of the 


\section{REVIEW ARTICLES}

best-performing neuroimaging journals and they supplemented relevant research outputs to the medical imaging field such as brain connectivity and pathological structural change of a patient's brain.

1. Frahm, J., Magnetic resonance functional neuroimaging: new insights into the human brain. Curr. Sci., 1999, 76, 735-743.

2. Jagannathan, N., Functional and pathophysiological study of disease processes in humans and animal systems: role of magnetic resonance imaging and in vivo MR spectroscopy. Curr. Sci., 2004, 86, 42-61.

3. Thomson Reuters, Whitepaper using bibliometrics: a guide to evaluating research performance with citation data; http://ipscience.thomsonreuters.com/m/pdfs $/ 325133$ thomson.pdf (accessed on 10 June 2016)

4. Prathap, G., A bibliometric profile of Current Science. Curr. Sci., 2014, 106, 958.

5. Zhang, L. and Feng, Y., Bibliometrics and visualization analysis of artificial blood vessel research. Curr. Sci., 2014, 106, 816-822.

6. Wang, J., Zheng, T., Wang, Q., Xu, B. and Wang, L., A bibliometric review of research trends on bioelectrochemical systems. Curr. Sci., 2015, 109, 2204.

7. Ho, Y.-S., A bibliometric analysis of highly cited articles in materials science. Curr. Sci., 2014, 107, 1565-1572.

8. Shahabuddin, S. M., Mapping neuroscience research in India - a bibliometric approach. Curr. Sci., 2013, 104, 1619-1626.

9. Van Eck, N. J., Waltman, L., van Raan, A. F., Klautz, R. J. and Peul, W. C., Citation analysis may severely underestimate the impact of clinical research as compared to basic research. PLoS ONE, 2013, 8, e62395.

10. Thomson Reuters, Journal Citation Reports. http://adminapps.webofknowledge.com/JCR/help/h toc.htm (accessed on 10 June 2016).

11. van Eck, N. J. and Waltman, L., Software survey: VOSviewer, a computer program for bibliometric mapping. Scientometrics, 2009, 84, 523-538.

12. van Eck, N. J., Waltman, L., Dekker, R. and van den Berg, J., A comparison of two techniques for bibliometric mapping: multidimensional scaling and VOS. J. Am. Soc. Inf. Sci. Technol., 2010, 61, 2405-2416.

13. Waltman, L., van Eck, N. J. and Noyons, E. C., A unified approach to mapping and clustering of bibliometric networks. $J$. Inf., 2010, 4, 629-635.

14. Van Eck, N. J. and Waltman, L., Text mining and visualization using VOSviewer. arXiv preprint 2011, arXiv:1109.2058.

15. Heersmink, R., van den Hoven, J., van Eck, N. J. and van den Berg, J., Bibliometric mapping of computer and information ethics. Ethics Inf. Technol., 2011, 13, 241-249.
16. Rodrigues, S., van Eck, N., Waltman, L. and Jansen, F., Mapping patient safety: a large-scale literature review using bibliometric visualisation techniques. BMJ Open, 2014, 4, e004468.

17. Waltman, L., van Raan, A. F. and Smart, S., Exploring the relationship between the engineering and physical sciences and the health and life sciences by advanced bibliometric methods. PLoS ONE, 2014, 9, e111530.

18. Jayaratne, Y. S. N. and Zwahlen, R. A., The evolution of dental journals from 2003 to 2012: a bibliometric analysis. PLOS ONE, 2015, 10, e0119503.

19. Lim, K. J. et al., Characteristics and trends of radiology research: a survey of original articles published in AJR and Radiology between 2001 and 2010. Radiology, 2012, 264, 796-802.

20. López-Abente, G. and Muñoz-Tinoco, C., Time trends in the impact factor of public health journals. BMC Public Health, 2005, $\mathbf{5}, 1$.

21. Rosenkrantz, A. B. and Ayoola, A., The impact factor of radiological journals: associations with journal content and other characteristics over a recent 12 -year period. Acad Radiol, in press, 23, 661-668.

22. Thomson Reuters, Scope notes. http://ip-science.thomsonreuters.com/mjl/scope/scope scie/\#RX (accessed on 10 June 2016).

23. Yeung, A. W. K., Tanabe, H. C., Suen, J. L. K. and Goto, T. K., Taste itensity modulates effective connectivity from the insular cortex to the thalamus in humans. NeuroImage, 2016, 135, 214222.

24. Seglen, P. O., Why the impact factor of journals should not be used for evaluating research. Br. Med. J., 1997, 314, 498.

25. Bergstrom, C. and West, J., About the eigenfactor project. http://www.eigenfactor.org/methods.htm (accessed on 10 June 2016).

26. Fellows, L. K., Heberlein, A. S., Morales, D. A., Shivde, G., Waller, S. and Wu, D., Method matters: an empirical study of impact in cognitive neuroscience. J Cogn. Neurosci., 2005, 17, $850-858$.

ACKNOWLEDGEMENTS. This work described was substantially supported by a grant from the Research Grants Council of the Hong Kong Special Administrative Region, China (HKU 766212M). The funding body had no role in the design of the study, and collection, analysis and interpretation of data as well as in writing the manuscript.

Received 19 June 2016; accepted 28 September 2016

doi: $10.18520 / \mathrm{cs} / \mathrm{v} 112 / \mathrm{i} 04 / 725-734$ 